\section{Molecular Syndromology}

Mol Syndromol 2018;9:287-294

DOI: $10.1159 / 000494451$
Accepted: August 27, 2018

by M. Schmid

Published online: November 13, 2018

\title{
Distal Arthrogryposis with Impaired \\ Proprioception and Touch: Description of an Early Phenotype in a Boy with Compound Heterozygosity of PIEZO2 Mutations and Review of the Literature
}

\author{
Jana Behunova ${ }^{a}$ Maria Gerykova Bujalkova ${ }^{a}$ Gabriel Gras ${ }^{b}$ Thomas Taylor ${ }^{a}$ \\ Ulrike Ihm ${ }^{\mathrm{a}}$ Susanne Kircher ${ }^{\mathrm{a}}$ Helga Rehder $^{\mathrm{a}}$ Franco Laccone $^{\mathrm{a}}$ \\ ${ }^{\mathrm{a}}$ Institute of Medical Genetics, Medical University of Vienna, and ${ }^{\mathrm{b}}$ VKKJ Center for Developmental Neurology and \\ Social Pediatrics, Vienna, Austria
}

\section{Keywords}

DAIPT · Distal arthrogryposis · Floppy infant · Hypotonia ·

PIEZO2 - Proprioception

\begin{abstract}
The recessive PIEZO2-associated disease, distal arthrogryposis with impaired proprioception and touch (DAIPT), is characterized by hypotonia, perinatal respiratory distress, significantly delayed motor milestones, and progressive symptoms of distal arthrogryposis and scoliosis. Here, we describe the youngest patient with DAIPT to date, who, at the age of 3.5 years, did not show a single clinical sign of distal arthrogryposis or contractures, but had a history of bilateral clubfoot operations. On the contrary, he presented with some features, not described thus far, such as syringohydromyelia, a small cyst of the spinal cord, moderate microcephaly with premature closure of anterior fontanelle, and spontaneous unilateral patella dislocation at the age of 32 months. Using whole exome sequencing, we identified 2 new different lossof-function mutations in the PIEZO2 gene in our patient. We also review the phenotypes of all 16 previously published
\end{abstract}

\section{KARGER}

(C) 2018 S. Karger AG, Basel

E-Mail karger@karger.com

www.karger.com/msy patients with DAIPT, summarize the distinctive clinical features of this rare genetic disorder, and recommend that DAIPT be included in the differential diagnosis of floppy infant. PIEZO2 is a unique ion channel that converts mechanical impulses into cellular signals and is involved in various mechanotransduction pathways. In addition to DAIPT, mutations in PIEZO2 have been described to cause 3 more distinct phenotypes of distal arthrogryposis, which are dominant and associated with gain-of-function mutations. On the contrary, recessive DAIPT is associated with loss-of-function PIEZO2 mutations.

(c) 2018 S. Karger AG, Basel

Distal arthrogryposis with impaired proprioception and touch (DAIPT; OMIM 617146) was first described in 2016 [Delle Vedove et al., 2016] in 10 patients from 4 consanguineous families that exhibited distal arthrogryposis, progressive scoliosis, significantly delayed motor milestones, severe hypotonia with muscle atrophy, and dysarthria along with normal intellectual development and transitory respiratory problems in early infancy. All pa-

Jana Behunova

Institute of Medical Genetics, Medical University of Vienna

Währinger Str. 10

AT-1090 Vienna (Austria)

E-Mail jana.behunova@meduniwien.ac.at 

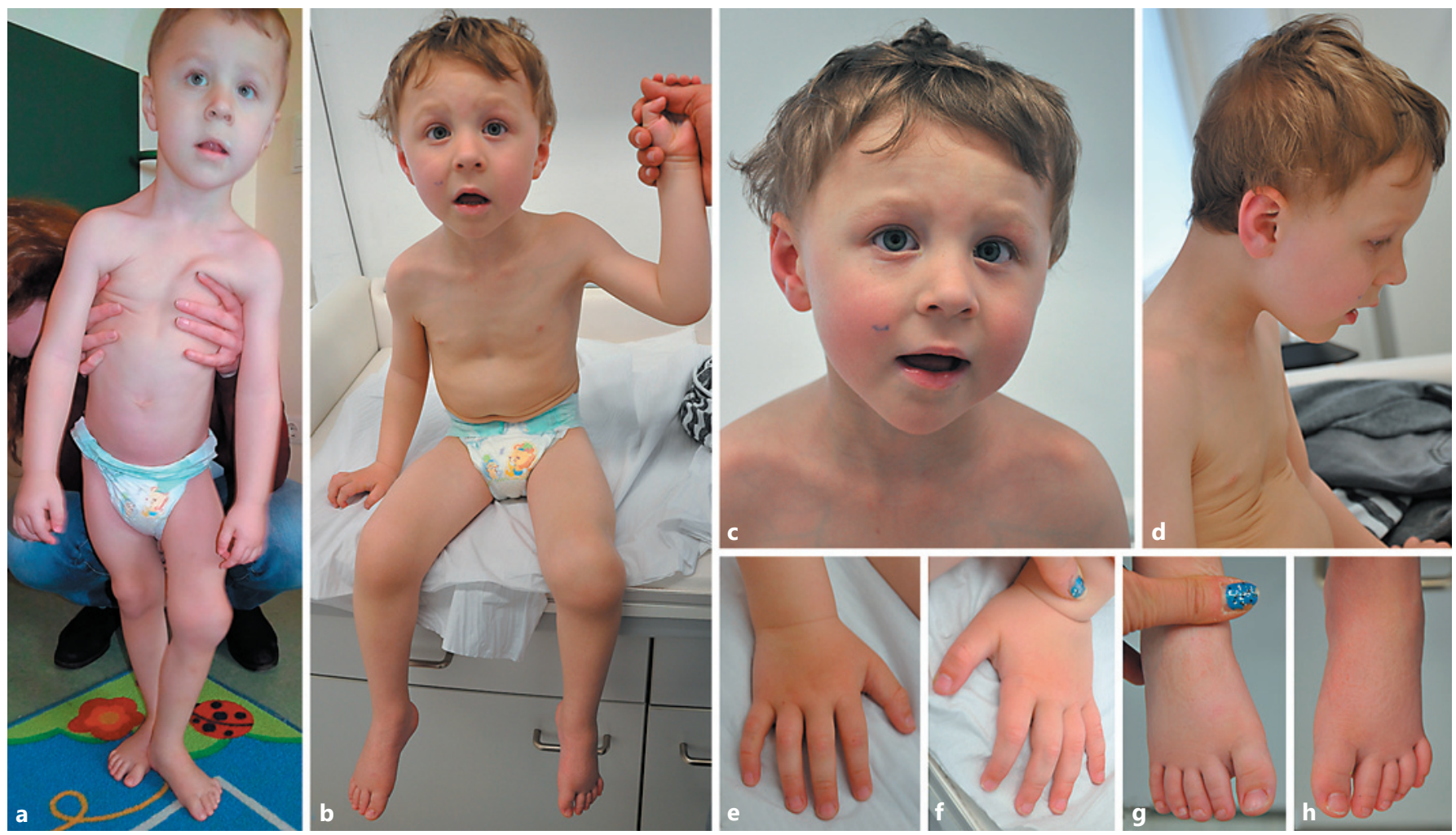

Fig. 1. Phenotype of the patient (age 3.5 years). a, b Whole body and extremities. Hypotonia, inability to stand or walk alone, and a dislocated left patella are shown. c, d Face and upper body. Slightly short palpebral fissures, pointed asymmetric chin, mild pectus excavatum, inverted nipples, and pronounced vascular drawing at the upper chest as well as loose skin at the lower chest are noticeable. e-h Hands and feet without contractures. Surgical correction of bilateral clubfeet at the age of 6 months was performed.

tients carried homozygous loss-of-function mutations in PIEZO2 (Piezo type mechanosensitive ion channel component 2; OMIM 613629). A wide range of sensory and kinematic functions was studied in 2 patients who both carried compound heterozygous mutations in the PIEZO2 gene [Chesler et al., 2016]. Mahmud et al. [2017] and Haliloglu et al. [2017] reported 4 more patients from 2 families. The heterozygous carriers (parents and siblings) were healthy. In contrast, the dominant PIEZO2 gain-offunction mutations result in distal arthrogryposis type 3 (DA3 or Gordon syndrome, with cleft palate, OMIM 114300), distal arthrogryposis type 5 (DA5 with eye anomalies, OMIM 108145), or Marden-Walker syndrome (OMIM 248700) [Coste et al., 2013; McMillin et al., 2014]. Here, we report the youngest patient with DAIPT who has been observed to date, revealing a distinct early phenotype with some new features and review the clinical characteristics of the DAIPT patients from the literature.

\section{Clinical Report}

A 3.5-year-old Caucasian male (born 2013) was referred to our Institute of Medical Genetics from the Center for Developmental Neurology and Social Pediatrics for genetic evaluation and diagnostics due to hypotonia with significantly delayed motor milestones.

The pregnancy was uncomplicated until the ultrasound organ screening (20th week of gestation) revealed bilateral pes equinovarus. Fetal MRI did not show any other additional pathologies. The mother mentioned that the fetal movements may have been reduced. Shortly after birth (40th week of gestation, $3,566 \mathrm{~g}, 52 \mathrm{~cm}$, OFC $33 \mathrm{~cm}$, and Apgar score 9/10/10), stridor and gasping were present as well as severe hypotonia, weakness, and cyanosis while feeding. The 1-week-old newborn had been described as "having a bell-shaped chest, almost without spontaneous breathing (vigorous stimulation was necessary), with very scarce spontaneous movements, even after a painful impulse." The boy required intensive care and oxygen support for 2 months and suffered pneumonia twice. A bronchoscopy did not reveal any structural anomalies in the respiratory airways, although almost complete closure of the airways in the hypopharynx and aditus laryngis regions was observed at the end of inspiration. Nasogastric tube feeding was given for the first 2 months, and the boy had significant diarrhea and reflux that later 


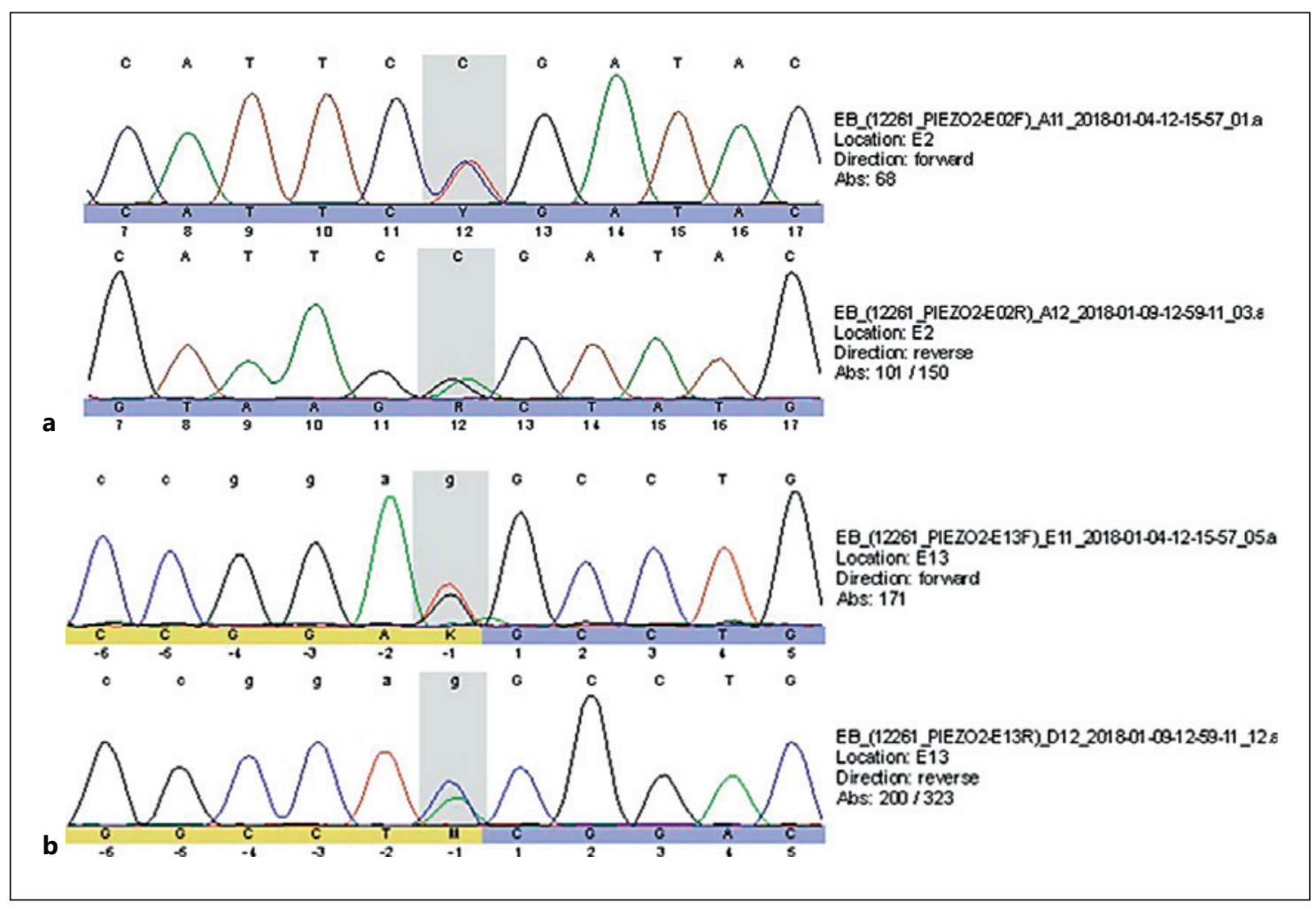

Fig. 2. Sequences of the patient's PIEZO2 mutations. a Mutation c.76C $>\mathrm{T}$ (p.Arg26Ter) in exon 2. b Mutation c.1528-1G $>\mathrm{T}$ in intron 12 .

improved. Early ultrasounds of brain, heart and abdomen were normal, as were the hearing test, ophthalmologic examination, metabolic evaluation, and array-CGH analysis. Two brain and spine MRIs were obtained at different times (the brain MRIs at the age of 1 month, while hospitalized at a neonatal intensive care unit, and at the age of 1 year and 6 months as a part of the neurological investigation; the spine MRIs in 1 year/2 months and 1 year/6 months). While no anomalies were observed in the brain (the microcephaly had not been mentioned, probably due to just mildly significant deviation), the spinal cord showed mild ectasia of the central canal in terms of syringohydromyelia and a small cystic lesion in the lower cervical spine, similar to a neurenteric or arachnoidal cyst, without signs of progression between imaging. Neurological evaluations showed hyporeflexia (11 months) and later areflexia (2 years) of the lower extremities, without pyramidal signs (Babinski, Oppenheim), which is not consistent with a diagnosis of neuromuscular disease or a disorder of the 2nd motoneuron. The boy never had seizures, and his EEG did not display any pathologies. His social development and eye contact were very good, although gross motor milestones were significantly delayed due to severe hypotonia; the patient could roll over at approximately 10 months, sit at 19 months, and crawl at 2 years/3 months, but he could not walk. His fine motor skill development was much better, and his speech and intellectual development were age appropriate. The patient was in the 50th percentile (P) for length and weight; however, OFC was slightly below P3, and the anterior fontanelle had closed prematurely (4 months). Surgery due to pes equinovarus had been performed at the age of 6 months on both feet after gypsum redress therapy. The patient also underwent a unilateral orchidopexy. Right-convex scoliosis with its apex at the thoracolumbar junction had been diagnosed at the age of 2 years, and the patient was treated with a back brace. At the age of 2 years/ 8 months, the boy woke up one morning with a painful swelling of his left knee without any known trauma. After an initial suspicion of juvenile arthritis, a spontaneous patella dislocation was diagnosed that later required surgical correction.

To date, the patient has no siblings, and his parents are healthy nonconsanguineous Caucasians. Physical examination at the age of 3.5 years showed a borderline low OFC (height $97.5 \mathrm{~cm} / \mathrm{P} 30$, weight $14 \mathrm{~kg} / \mathrm{P} 24$, and OFC $47 \mathrm{~cm} /$ just below P3). The patient could sit, crawl, and play on the ground but was unable to stand independently (Fig. 1a). Spoken language was logical and in complete sentences, although his speech was nasal, slurred, and sometimes difficult to understand. He showed mild pectus excavatum (Fig. 1b, d), scoliosis, scapulae alatae, and significant global hypotonia. The joints were moderately hypermobile and the left knee was swollen with a laterally dislocated patella (Fig. 1a, b). The extremities were otherwise symmetric, without contractures or any other pathologies, except for mildly flat feet (Fig. 1e-h). His skin vascular drawing of the upper part of the chest and face was more pronounced, whereas redundant skin folds were observed on the chest and abdomen (Fig. 1c, d). The nipples were widely spaced, small, and anteverted. There were no abdominal hernias; the liver and spleen were not palpable, and the genitals were age appropriate. Some subtle dysmorphic craniofacial features were observed 
(Fig. 1b-d), including a flat and asymmetric occiput and a broad forehead, in contrast to a pointed and somewhat asymmetrical chin. The ears were borderline low set but well formed, and the eyes were slightly deeper set, had an almond-round shape, and had short palpebral fissures and epicanthus. The philtrum was long, and the palate was high. The teeth and neck showed no abnormalities. At the time of the last counseling, the parents mentioned that the boy ( 4 years/ 3 months) still could not walk.

\section{Materials and Methods}

A whole exome sequencing library was prepared with the TruSeq Exome Kit (Illumina, San Diego, CA, USA) and further analyzed using a NextSeq 500 Sequencing System (Illumina). Bioinformatics evaluation was performed using the VarSeq Software (Golden Helix, Bozeman, MT, USA), and $94 \%$ of the analyzed sequences were covered $>20$ times. Various databases (ExAC, EVS, 1000 Genomes, HGMD, and OMIM) were used for the estimation of variant frequencies and/or their impact. Variants were filtered according to their possible association with the patient's phenotype, and where necessary, their pathogenicity was assessed with 6 prediction programs (SIFT, Polyphen2, MutationTaster, MutationAssessor, FATHMM, and FATHMM MKL Coding). All relevant mutations were confirmed by Sanger sequencing.

\section{Results}

Through whole exome sequencing analysis, 2 compound heterozygous mutations were identified in the patient's PIEZO2 gene, both of which were predicted to cause loss of function at the protein level (Fig. 2a, b). The first mutation, c.76C > T (NM_022068.3), is located in exon 2 and is predicted to produce a premature stop codon, p.Arg26Ter (NP_071351.2). It was not listed in ExAC and had a frequency of 0.0002 in 1000 Genomes. The second mutation, c.1528-1G $>\mathrm{T}$, is localized in a highly conserved dinucleotide of the acceptor splice site in intron 12 and is predicted to affect splicing. It was not listed in ExAC or 1000 Genomes. Each parent was shown to carry 1 heterozygous mutation by Sanger sequencing (c.76C $>\mathrm{T}$ in the mother and c.1528-1G $>\mathrm{T}$ in the father). The most important phenotypic features of DAIPT based on the reported 16 patients from 8 families and our patient are listed in Tables 1, 2.

\section{Discussion}

The PIEZO2 gene is located in chromosome 18 (18p11.22), its size is $477 \mathrm{~kb}$, and it comprises 52 exons. The size of themaingenetranscript(ENST00000503781.7)
Table 1. Clinical features of DAIPT

Patients, $n(\%)$

$\begin{array}{lc}\text { Main features } & \\ \text { Hypotonia } & 15 / 15(100) \\ \text { Motor delay } & 17 / 17(100) \\ \text { Significant walking delay ( } \geq 5 \text { years of age) } & 15 / 15(100) \\ \text { Absent deep tendon reflexes } & 15 / 15(100) \\ \text { Progressive scoliosis } & 16 / 17(94) \\ \text { Bilateral pes equinovarus/other foot deformity } & 13 / 15(87) \\ \text { Camptodactyly } & 11 / 15(73) \\ \text { Thumb deformity } & 13 / 16(81) \\ \text { Short stature } & 11 / 14(79) \\ \text { Neonatal respiratory distress with or without stridor } & 7 / 8(88) \\ \text { Dysarthria/nasal speech } & 9 / 9(100) \\ \text { Reduced proprioception/vibratory sense } & 7 / 7(100) \\ \text { Axonal sensory neuropathy } & 5 / 5(100) \\ \text { Minor features } & \\ \text { Early feeding difficulties } & 5 / 12(42) \\ \text { Hypomimia/ptosis } & 6 / 13(46) \\ \text { Transitory/mildly elevated CK } & 3 / 6(50) \\ \text { Congenital hip dysplasia } & 3 / 4(75) \\ \text { Arachnodactyly } & 9 / 16(56)\end{array}$

CK, creatine kinase; DAIPT, distal arthrogryposis with impaired proprioception and touch. For further details, see Table 2.

is $8,259 \mathrm{bp}$, and the encoded protein consists of 2,752 amino acids. The mutations in PIEZO2 can be either of a gain-of-function type, causing dominant phenotypes of distal arthrogryposis, or of a loss-of-function type, causing recessive DAIPT. The majority of PIEZO2 dominant mutations are clustered in 2 regions of the gene that encode the C-terminal domain and appear to be mutation hotspots, particularly the last exon 52. In contrast, the mutations in recessive DAIPT do not show any locus predilection, as they are distributed throughout the entire gene [Chesler et al., 2016; Delle Vedove et al., 2016; Haliloglu et al., 2017; Mahmud et al., 2017]. The PIEZO2 protein contains more than 30 transmembrane domains and functions as part of mechanically activated cation channels [Coste et al., 2010]. The only other known protein with similar mechanotransduction function in humans, PIEZO1, is coded by the PIEZO1 gene, which shares approximately $50 \%$ of its DNA sequence with PIEZO2. PIEZO1 also shows dominant gain-of-function mutations and recessive loss-of-function mutations that cause different phenotypes, namely dehydrated hereditary stomatocytosis with hemolytic anemia (dominant) and generalized lymphatic dysplasia (recessive), but not arthrogryposis [Zarychanski et al., 2012; Li et al., 2014; Fotiou et al., 2015]. PIEZO2 and PIEZO1 are very large, complex, and conserved transmembrane proteins [Coste et al., 2010]. They are also termed stretch-activated channels and are present in a variety of cell types in which me-
Behunova et al. 
₹

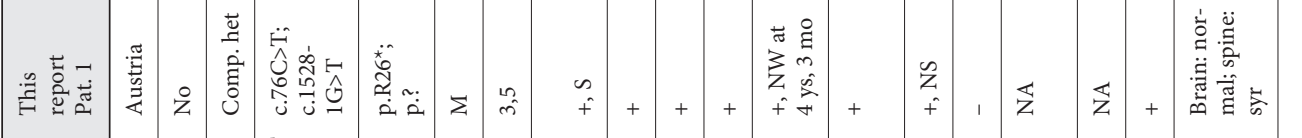

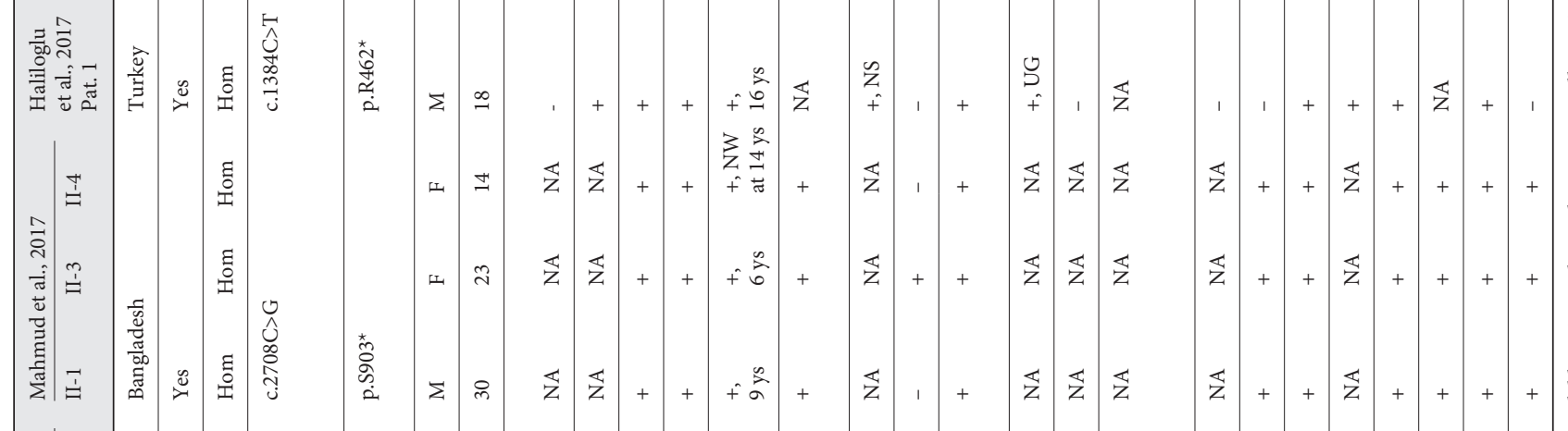

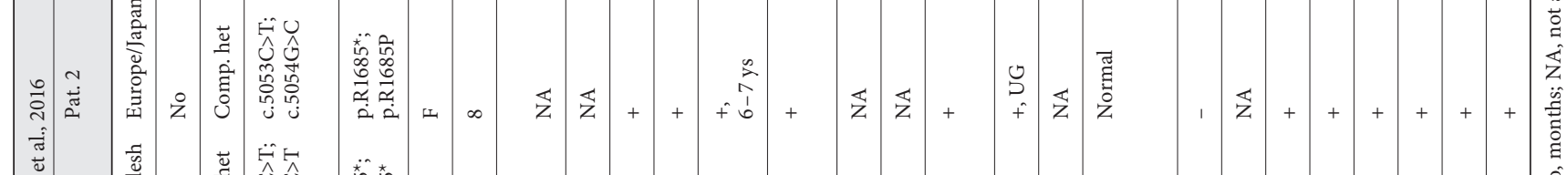

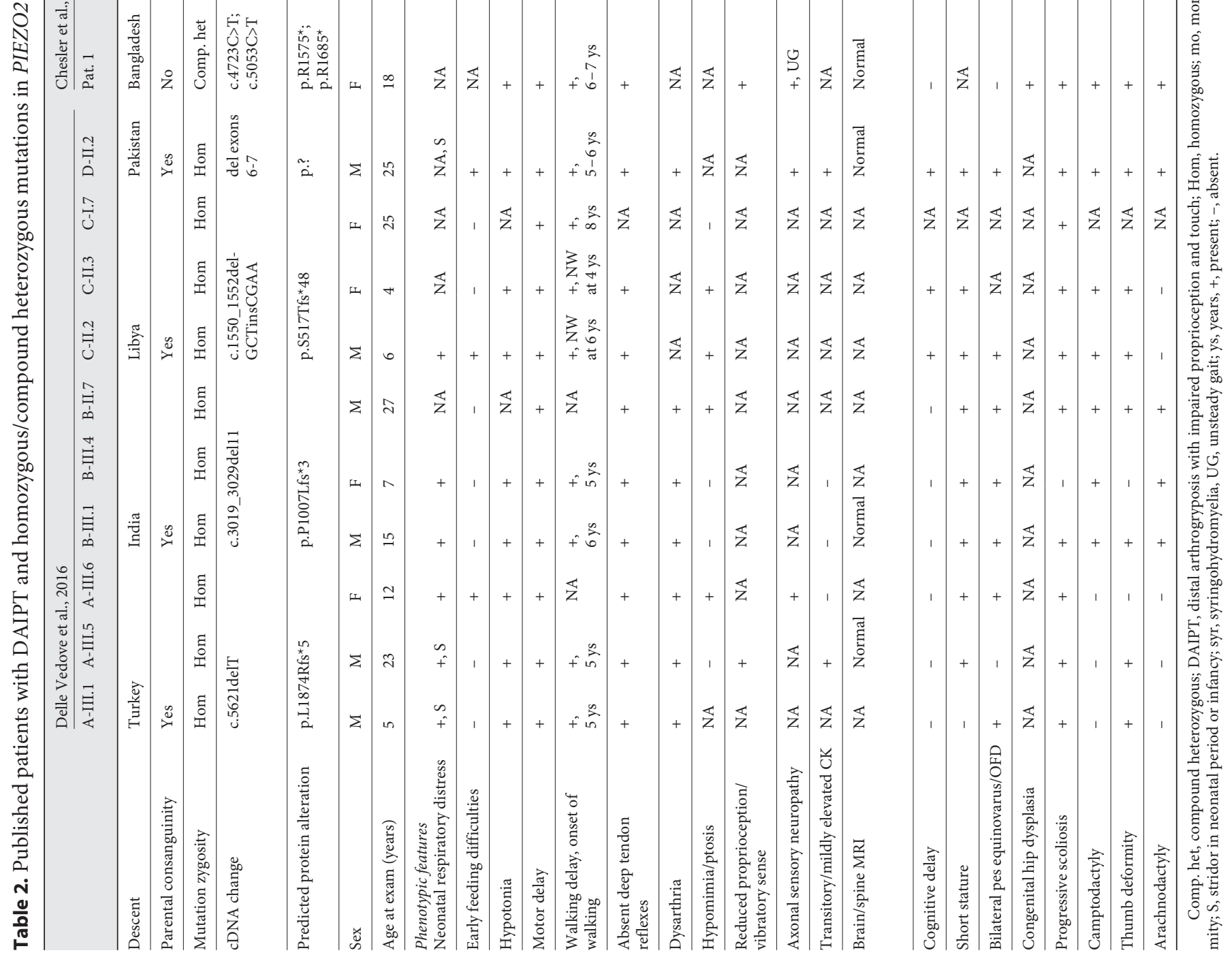


chanical stimuli need to be transduced into electric signals, such as in somatosensory neurons, muscle cells, osteoblasts, endothelial cells, and inner ear sensory cells [Coste et al., 2010; Sachs, 2010; Pardo-Pastor et al., 2018]. In humans, PIEZO2 is the major mechanotransducer for proprioceptors [Woo et al., 2015]. Proprioception, the perception of body and limb position, is mediated by specialized mechanosensory neurons that convey information about the stretch and tension experienced by muscles, tendons, skin, and joints. The loss-of-function and gain-of-function mutations are consistent with the musculoskeletal symptomatology in PIEZO2-associated diseases, where both reduced and enhanced protein activity of PIEZO2 have diverse negative effects. PIEZO2 is also a versatile airway stretch sensor of lung inflation, and it is critical for proper lung expansion, establishing efficient respiration at birth and maintaining normal breathing in adult mice [Nonomura et al., 2017]. These data are compatible with human PIEZO2-associated phenotypes in respect to respiratory distress in the neonatal period in DAIPT patients as well as with regard to the progressive restrictive lung disease in adults with DA5.

Biallelic loss-of-function mutations in PIEZO2 cause a specific DAIPT phenotype, including severe hypotonia with significant delay of motor milestones, transient respiratory distress and feeding problems in early infancy as well as symptoms of severe progressive scoliosis and progressive contracture deformities of the hands and feet (distal arthrogryposis). Comparing the features of recessive and dominant PIEZO2-associated diseases, distal arthrogryposis in the dominant forms is typically congenital, whereas scoliosis is less frequent, reported in only 16 of 61 patients [McMillin et al., 2014]. Respiratory and feeding problems in infancy have not yet been described in dominant PIEZO2-associated diseases, but progressive restrictive lung disease in adults has been observed in DA5. Intellectual development is normal also in dominant PIEZO2-associated diseases, with the exception of the most severe and rare form, the Marden-Walker syndrome, which apart from joint contractures and kyphoscoliosis includes many additional anomalies (mask-like face, blepharophimosis, micrognathia, cleft palate, chest deformities, failure to thrive, and renal and cerebral anomalies). However, to date, only a single patient with Marden-Walker syndrome has been confirmed to carry a dominant PIEZO2 mutation [McMillin et al., 2014]. DA3 (Gordon syndrome) involves an additional cleft palate or a bifid uvula. Patients with DA5 also manifest various ocular symptoms (e.g., ophthalmoplegia, ptosis, deep-set eyes, blepharophimosis, and retinal abnormalities) and progressive restrictive lung disease [McMillin et al., 2014; Alisch et al., 2017]. However, some features of dominant PIEZO2-associated diseases overlap. Some brain anomalies described in Marden-Walker syndrome (DandyWalker or Arnold-Chiari malformation) have also been incidentally noted in both DA3 and DA5 [McMillin et al., 2014].

The following specification of a DAIPT phenotype is based on 16 so far published patients plus our patient and includes severe hypotonia with motor delay and normal intelligence, severe scoliosis, progressive distal arthrogryposis, and some other features (Table 1,2). Distal arthrogryposis in patients with DAIPT has been observed as "congenital," particularly regarding bilateral foot deformities [Delle Vedove et al., 2016]. Contracture deformities of the upper limbs were present in 15 of the 16 previously reported patients. Only our patient at the age of 3.5 years as well as a 12-year-old patient of Delle Vedove et al. [2016] did not manifest any hand contractures. However, the age of onset of hand contractures has been rarely specified; only the report by Mahmoud et al. [2017] described the earliest, but not congenital, case of both hand and foot contractures at the age of 6 months. The bilateral clubfeet in our patient had been diagnosed prenatally, but no finger, toe, or wrist contractures were present at the age of 3.5 years. In contrast, congenital joint contractures, including the upper limbs, are typical in patients with dominant PIEZO2 mutations or other types of distal arthrogryposis, e.g., due to mutations in $M Y H 3$, MYH8, TPM2, TNNI2, TNNT3, MYBPC1, ECEL1, or FBN2. Most of these genes encode structural components of the contractile apparatus of fast-twitch myofibers: MYH3 encodes embryonic myosin, TNNI2 troponin I, TNNT3 troponin T, TPM2 tropomyosin 2, and perinatal skeletal myosin heavy chain 8 (MYH8) [Toydemir et al., 2006a, b; Sung et al., 2003a, b]. The MYBPC1 gene encodes myosin-binding protein $\mathrm{C}$, slow type; ECEL1 encodes one of the zinc metalloproteases expressed during embryogenesis in CNS as well as in skeletal muscle, and FBN2 encodes fibrillin-2, a part of microfibrils of elastic fibers important for early elastogenesis in skin, ligaments, muscles, and other tissues [Putnam et al., 1995; Gurnett et al., 2010; Dieterich et al., 2013]. Early and progressive scoliosis (beginning between 1 and 5 years of age) was a significant health problem noted in all DAIPT patients sometimes even requiring surgery. Almost $80 \%$ of the patients $(11 / 14)$ had short stature. More than half of the patients $(9 / 16)$ manifested arachnodactyly. The majority of DAIPT patients $(11 / 15)$ gained the ability to walk, although significantly delayed (between 5 and 16 years). An
292

Mol Syndromol 2018;9:287-294 DOI: $10.1159 / 000494451$
Behunova et al. 
unsteady, wide-based gait is described, only possible with opened eyes. Deep tendon reflexes were missing in all patients. Brain and spinal cord MRI were normal, only our patient had mild syringohydromyelia and a small cystic lesion in the lower C-spine without progression. All patients who underwent nerve conduction velocity or proprioception testing (5/5 and $7 / 7$, respectively) showed mild sensory polyneuropathy and/or impaired proprioception. Some individuals (3/6) had transiently mildly elevated creatine kinase. There were no particular ophthalmological problems and no cleft palate compared to patients with dominant distal arthrogryposis due to PIEZO2 mutations. However, some patients with DAIPT had a high palate and mild ptosis or hypomimia. Cognitive development was usually normal. Only in one family, mild cognitive disability had been described in 2 affected children, possibly influenced also by a low socioeconomic status of the family [Delle Vedove et al., 2016]. Heterozygous carriers of PIEZO2 loss-of-function mutations (parents and siblings) were healthy, without any signs of neuromuscular or skeletal symptomatology.

In our patient, we observed a few features that have not yet been reported in DAIPT, such as borderline microcephaly with premature closure of the anterior fontanelle and head asymmetry, mild syringohydromyelia with a small cyst, spontaneous left patella dislocation at the age of 2 years 8 months, without any trauma, and cryptorchidism. No other patient had shown an anomaly of the spinal cord via MRI and assessment of whether the syringomyelia observed in our patient is related to the PIEZO2 mutations is currently not possible.

In conclusion, we assume that at an early age the DAIPT phenotype may be rather unspecific, as it is characterized by a clinical pattern of floppy infant with respiratory distress and bilateral foot deformities. These features could manifest in various monogene disorders (e.g., nemaline myopathies, fetal akinesia sequence, lethal congenital contractures, distal spinal muscular atrophy 1, and others). The typical signs of hand and finger contractures in DAIPT probably develop at a later age, as does scoliosis. The motor and cognitive development cannot be assessed in early infancy as well. Thus, it may be possible that most very young DAIPT patients will remain undiagnosed, which may hamper early management of scoliosis as well as notification of the family about the $25 \%$ recurrence risk. We recommend that DAIPT be included in the differential diagnosis of floppy infant, particularly in the presence of additional foot deformities and respiratory distress. Considering the lack of clinical specificity for DAIPT at a young age and the large size of the PIEZO2 gene, whole exome sequencing after exclusion of the most common genetic causes of severe neonatal hypotonia (i.e., chromosomal anomalies, spinal muscular atrophy, Prader-Willi syndrome, and myotonic dystrophy type 1) is the most effective diagnostic method for DAIPT in early childhood.

\section{Acknowledgments}

We thank the family for the willingness to participate in this report. We also thank Marion Hagl for technical assistance.

\section{Statement of Ethics}

Informed consent was obtained prior to genetic analyses and to publish clinical details of this study. The authors have no ethical conflicts to disclose.

\section{Disclosure Statement}

The authors declare no conflicts of interest.

\section{References}

Alisch F, Weichert A, Kalche K, Paradiso V, Longardt AC, et al: Familial Gordon syndrome associated with a PIEZO2 mutation. Am J Med Genet A 173:254-259 (2017).

Chesler AT, Szczot M, Bharucha-Goebel D, Čeko $\mathrm{M}$, Donkervoort S, et al: The role of PIEZO2 in human mechanosensation. N Engl J Med 375:1355-1364 (2016).

Coste B, Mathur J, Schmidt M, Earley TJ, Ranade S, et al: Piezol and Piezo2 are essential components of distinct mechanically activated cation channels. Science 330:55-60 (2010).

PIEZO2, Early Recessive Phenotype

\footnotetext{
Coste B, Houge G, Murray MF, Stitziel N, Bandell $\mathrm{M}$, et al: Gain-of-function mutations in the mechanically activated ion channel PIEZO2 cause a subtype of distal arthrogryposis. Proc Natl Acad Sci USA 110:4667-4672 (2013).

Delle Vedove A, Storbeck M, Heller R, Hölker I, Hebbar M, et al: Biallelic loss of proprioception-related PIEZO2 causes muscular atrophy with perinatal respiratory distress, arthrogryposis, and scoliosis. Am J Hum Genet 99:1206-1216 (2016).

Dieterich K, Quijano-Roy S, Monnier N, Zhou J, Fauré J, et al: The neuronal endopeptidase ECEL1 is associated with a distinct form of recessive distal arthrogryposis. Hum Mol Genet 22:1483-1492 (2013).

Fotiou E, Martin-Almedina S, Simpson MA, Lin $S$, Gordon $\mathrm{K}$, et al: Novel mutations in PIEZO1 cause an autosomal recessive generalized lymphatic dysplasia with non-immune hydrops fetalis. Nat Commun 6:8085 (2015).
} 
Gurnett CA, Desruisseau DM, McCall K, Choi R, Meyer ZI, et al: Myosin binding protein $\mathrm{Cl}$ : a novel gene for autosomal dominant distal arthrogryposis type 1. Hum Mol Genet 19: 1165-1173 (2010).

Haliloglu G, Becker K, Temucin C, Talim B, Küçükşahin N, et al: Recessive PIEZO2 stop mutation causes distal arthrogryposis with distal muscle weakness, scoliosis and proprioception defects. J Hum Genet 62:497-501 (2017).

Li J, Hou B, Tumova S, Muraki K, Bruns A, et al: Piezo1 integration of vascular architecture with physiological force. Nature 515:279-282 (2014).

-Mahmud AA, Nahid NA, Nassif C, Sayeed MS, Ahmed MU, et al: Loss of the proprioception and touch sensation channel PIEZO2 in siblings with a progressive form of contractures. Clin Genet 91:470-475 (2017).

McMillin MJ, Beck AE, Chong JX, Shively KM, Buckingham KJ, et al: Mutations in PIEZO2 cause Gordon syndrome, Marden-Walker syndrome, and distal arthrogryposis type 5 . Am J Hum Genet 94:734-744 (2014).
Nonomura K, Woo SH, Chang RB, Gillich A, Qiu $\mathrm{Z}$, et al: Piezo2 senses airway stretch and mediates lung inflation-induced apnoea. Nature 541:176-181 (2017).

-Pardo-Pastor C, Rubio-Moscardo F, VogelGonzález M, Serra SA, Afthinos A, et al: Piezo2 channel regulates RhoA and actin cytoskeleton to promote cell mechanobiological responses. Proc Natl Acad Sci USA 115:19251930 (2018).

Putnam EA, Zhang H, Ramirez F, Milewicz DM: Fibrillin-2 (FBN2) mutations result in the Marfan-like disorder, congenital contractural arachnodactyly. Nat Genet 11:456-458 (1995).

Sachs F: Stretch-activated ion channels: what are they? Physiology (Bethesda) 25:50-56 (2010).

-Sung SS, Brassington AME, Grannatt K, Rutherford A, Whitby FG, Krakowiak PA, et al: Mutations in genes encoding fast-twitch contractile proteins cause distal arthrogryposis syndromes. Am J Hum Genet 72:681-690 (2003a).
Sung SS, Brassington AM, Krakowiak PA, Carey JC, Jorde LB, Bamshad M: Mutations in TNNT3 cause multiple congenital contractures: a second locus for distal arthrogryposis type 2B. Am J Hum Genet 73:212-214 (2003b).

Toydemir RM, Rutherford A, Whitby FG, Jorde LB, Carey JC, Bamshad MJ: Mutations in embryonic myosin heavy chain $(M Y H 3)$ cause Freeman-Sheldon syndrome and SheldonHall syndrome. Nat Genet 38:561-565 (2006a).

Toydemir RM, Chen H, Proud VK, Martin R, van Bokhoven H, et al: Trismus-pseudocamptodactyly syndrome is caused by recurrent mutation of MYH8. Am J Med Genet 140:23872393 (2006b).

-Woo SH, Lukacs V, de Nooij JC, Zaytseva D, Criddle CR, et al: Piezo2 is the principal mechanotransduction channel for proprioception. Nat Neurosci 18:1756-1762 (2015).

Zarychanski R, Schulz VP, Houston BL, Maksimova Y, Houston DS, et al: Mutations in the mechanotransduction protein PIEZO1 are associated with hereditary xerocytosis. Blood 120:1908-1915 (2012) 